JHR

36,4

738

Received 3 August 2020

Revised 24 September 2020 2 November 2020

Accepted 3 November 2020

\section{Vulnerability toward the development of diabetes mellitus type 2 among adults from a rural community of West Bengal, India}

\author{
Shibaji Gupta \\ Department of Community Medicine, Midnapore Medical College, \\ Midnapore, India, and \\ Arup Chakraborty \\ Department of Community Medicine, Medical College and Hospital Kolkata, \\ Kolkata, India
}

\begin{abstract}
Purpose - India has over half a million diabetics, with many others at risk. The Indian Diabetes Risk Score (IDRS) is a simple and validated tool used for mass screening of diabetes mellitus type 2 at the community level. This study assessed the vulnerability of developing diabetes in adults of a rural community of West Bengal using the IDRS and finds out the relationship of the risk of developing diabetes with socioclinical variables. Design/methodology/approach - Multi-stage sampling was employed to select one eligible nondiabetic adult from selected families residing in the rural field practice area of a medical college in West Bengal. They were interviewed with a predesigned and pretested data collection schedule and examined.

Findings - Among 197 participants, $83.8 \%$ were female, $51.8 \%$ were illiterate and $57.4 \%$ came from Class IV of Prasad's socioeconomic scale. Of participants, $22.8 \%$ had existing known morbidities, and $23.9 \%$ had some form of substance addiction. In total, $46.8 \%$ of the participants on whom the IDRS could be applied $(n=175)$ were at high risk of developing diabetes $($ Score $=60)$. Gender and existing comorbidities significantly predicted a high risk of diabetes.

Originality/value - A large proportion of the Indian population yet to be diagnosed with diabetes are at a high risk of having the disease. Early detection of the disease can help curtail its complications and reduce its clinical, social and economic burden. Mass screening tools like the IDRS thus become a very important tool in India's attempts to fight diabetes.
\end{abstract}

Keywords Community based, Diabetes, Indian diabetes risk score, India

Paper type Research paper

\section{Introduction}

The number of diabetics around the world is expected to increase from 366 million cases in 2011 to 552 million cases by 2030 as per the International Diabetes Federation (IDF) if no suitable urgent action is taken [1].

India is often quoted as the Diabetes Capital of the World, besides China. Every fifth diabetic across the globe is an Indian, and the total burden of morbidity in India is expected to reach 100 million cases by 2030 . Added to this increasing burden, the problem is compounded by the fact that $66 \%$ of Indians who are diabetic go undiagnosed compared to $50 \%$ in Europe and $33 \%$ in the USA [2].

(C) Shibaji Gupta and Arup Chakraborty. Published in Journal of Health Research. Published by Emerald Publishing Limited. This article is published under the Creative Commons Attribution (CC BY 4.0) licence. Anyone may reproduce, distribute, translate and create derivative works of this article (for both commercial and non-commercial purposes), subject to full attribution to the original publication and authors. The full terms of this licence may be seen at http://creativecommons.org/licences/by/4.0/legalcode

Source of funding: Self-funded.
Journal of Health Research Vol. 36 No. 4,2022 pp. $738-745$

Emerald Publishing Limited e-ISSN: 2586-940X DOI 10.1108/JHR-08-2020-0320 
Responding to the alarming rise in diabetics, the Government of India has laid special emphasis on this noncommunicable disease in its National Health Policy, and the National Program for Prevention and Control of Cancer, Diabetes, Cardiovascular Diseases and Stroke (NPCDCS) has been designed to address such concerns [3].

Most diabetics are aged between 30-40 years, which has a significant impact on the social and economic cost of the country [4]. Early detection of diabetes by screening methods with a special focus on the population at higher risk of developing the disease can help delay or intercept its complications and thereby reduce the clinical, social and economic burden due to diabetes [5].

The present study aimed to assess the vulnerability among adults of a rural community of West Bengal toward developing diabetes mellitus type 2, using the Indian Diabetes Risk Score (IDRS). The IDRS, developed by V Mohan, one of India's leading diabetologists, from the Madras Diabetes Research Foundation, is a validated, simple, low cost, feasible tool for mass screening of diabetes mellitus type 2 at the community level, which has been used by many studies across India [2, 6-9].

\section{Methods}

The present descriptive, epidemiological study with a cross-sectional design was conducted in the rural field practice area of a medical college of West Bengal over a period of three months (May-July 2019).

A predesigned and pretested schedule was used to collect data, which had questions on socioclinical details and questions based on the IDRS. Waist circumference was measured in centimeters at the level of the umbilicus, using a nonstretchable measuring tape with the nearest calibration of $1 \mathrm{~mm}$.

The IDRS used is given below [2]:

\begin{tabular}{llr}
\hline Risk factor & Category & Score \\
\hline \multirow{2}{*}{ Age (years) } & $<35$ & 0 \\
& $35-49$ & 20 \\
& $>49$ & 30 \\
Abdominal obesity (in cm) & $<80(\mathrm{f}) ; 90(\mathrm{~m})$ & 0 \\
(f) = female & $80-89(\mathrm{f}) ; 90-99(\mathrm{~m})$ & 10 \\
(m) = male & $>=90(\mathrm{f}) ;>=100(\mathrm{~m})$ & 20 \\
Physical activity & Vigorous exercise or strenuous at work & 0 \\
& Moderate exercise at work/home & 10 \\
& Mild exercise at work/home & 20 \\
Family history of diabetes & No exercise and sedentary at work/home & 30 \\
& Nondiabetic & 0 \\
Total & One parent diabetic & 10 \\
& Both parents' diabetic & 20
\end{tabular}

Note(s): Total score: $>=60$ : high risk, 30-50: medium risk, $<30$ : low risk

A total of 227 line-listed families were residing in the defined area. A similar Indian study completed on the rural population found that $12.1 \%$ of its participants had a high risk of diabetes [10]. The sample size was calculated using the formula $n_{0}=z^{2} p q / e^{2}$ and was further corrected, since the population was finite, using the formula $n=n_{0} /\left[1+\left\{\left(n_{0}-1\right) / N\right\}\right][11]$. Here, $n_{0}=$ sample size before correction, $z^{2}=3.84 \approx 4$ (at 95\% confidence interval), prevalence $(p)=12.1 \%, N=$ total number of line listed families $=227, q=1-p$ and $e=20 \%$ of $p$, sample size $(n)$ was calculated to be 173 . Assuming a nonresponse rate of $20 \%$, considering 
JHR

36,4

740

the fact that some families would either be unwilling to participate or were unavailable and some respondents would not be aware of the status of diabetes in their parents, the sample size was finally calculated to be 208 . Hence, 208 families were randomly selected from the list of all available families. In total, 197 families were finally included in the study, as the rest were either unwilling to participate or were unavailable after three visits on three consecutive days of data collection. Nonseriously ill adult members (aged more than 18 years) present at the time of data collection, not known to be diabetic, and willing to participate in the study, were considered eligible. One eligible member from each of these families was selected by simple random sampling for interview and waist circumference measurement.

Data were entered and analyzed using Statistical Packages for Social Science (SPSS) ${ }^{\circledR}$ (SPSS Inc, Chicago, IL, USA) version 16.0. Results were obtained based on the predefined objectives as ratios and proportions. Sociodemographic variables found to be showing significant statistical predictability toward a high risk of diabetes mellitus type 2 (as per IDRS score) by univariate logistic regression analysis were further assessed using multivariable logistic regression.

\section{Ethical consideration}

The study proposal was placed before the Institute's Ethics Committee of the concerned medical college, and permission was obtained (Ref. No. MC/Kol/IEC/Non-spon/364/04/19 dated 27.4.2019). The participants were included only after informed consent, and their identities were not disclosed. The waist circumference of the female subjects was measured in the presence of the local Accredited Social Health Activist (ASHA) worker or another female of the household if the study subject was female.

\section{Results}

A total of 197 participants were included in the present study, with a mean age of 42.10 $( \pm 22.86)$ years, out of whom $83.8 \%(n=165)$ were female and $53.8 \%(n=106)$ were Hindus. The majority of the study subjects were illiterate $(51.8 \% ; n=102)$ and presently married $(76.6 \% ; n=151)$. Of participants, $57.4 \%(n=113)$ belonged to socioeconomic Class IV as per the BG Prasad scale of socioeconomic status, modified in July 2018 - the BG Prasad scale classifies a family based on monthly per capita income into one of five socioeconomic classes (Class I to V) - with Class I denoting the highest and Class V denoting the lowest socioeconomic class [12]. In total, $22.8 \%(n=45)$ had known comorbidities like hypertension, hypothyroidism, gout; $23.9 \%(n=47)$ were addicted to at least one substance of addiction such as tobacco smoking, smokeless tobacco or alcohol. The sociodemographic characteristics of the study participants are given in Table 1.

Participants aware of the status of diabetes in their parents $(n=175)$ were evaluated based on the IDRS. Of them, most of the participants $(49.7 \% ; n=87)$ were aged between $35-49$ years with a score of $20 ; 54.3 \%(n=95)$ of whose parents were nondiabetic, scoring zero (0). For physical activity, most $(41.7 \% ; n=73)$ were classified in the category of "mild exercise at work/home" with a score of 20 , while $46.9 \%(n=82)$ had a waist circumference of more than $90 \mathrm{~cm}$ (for females) and $100 \mathrm{~cm}$ (for males), scoring 20 points. In total, $46.8 \%$ $(n=82)$ of the participants were at high risk (score: $>=60), 38.8 \%(n=68)$ were at medium risk (score: $30-50)$ and the rest $(14.4 \% ; n=25)$ were at low risk of developing diabetes mellitus type 2 (score: $<30$ ), as per their corresponding IDRS (Table 2).

Gender $(\phi=0.007)$, addiction $(\phi=0.060)$ and presence of known comorbidities $(\phi=0.002)$ individually predicted significantly for high risk of developing diabetes mellitus type 2 in univariate logistic regression, while the results with other sociodemographic variables under consideration were not found significant. These three variables were subjected to multivariable logistic regression, and gender [adjusted odds ratio, i.e. $\mathrm{AOR}=2.737$ 


\begin{tabular}{|c|c|c|c|}
\hline Socio-clinical characteristics & Category/Group & No. $(\%)$ & Vulnerability \\
\hline \multirow[t]{2}{*}{ Gender } & Male & $32(16.2)$ & developing \\
\hline & Female & $165(83.8)$ & \\
\hline \multirow{2}{*}{ Religion } & Hinduism & $106(53.8)$ & diabete \\
\hline & Islam & $91(46.2)$ & \\
\hline \multirow[t]{4}{*}{ Educational qualification } & Illiterate & $102(51.8)$ & \\
\hline & Primary & 27 (13.7) & 741 \\
\hline & Middle & $47(23.9)$ & \\
\hline & Secondary and above & $21(10.6)$ & \\
\hline \multirow[t]{4}{*}{ Marital status } & Presently married & $151(76.6)$ & \\
\hline & Unmarried & $6(3.1)$ & \\
\hline & Widowed & $28(14.2)$ & \\
\hline & Separated & $12(6.1)$ & \\
\hline \multirow{5}{*}{ Socioeconomic status* with per capita monthly income (INR) } & Class I (7008 or more) & $0(0.0)$ & \\
\hline & Class II (3504-7007) & $0(0.0)$ & \\
\hline & Class III (2102-3503) & $5(2.5)$ & \\
\hline & Class IV (1051-2101) & $113(57.4)$ & \\
\hline & Class V (1050 or less) & 79 (40.1) & $\begin{array}{l}\text { Table } 1 . \\
\text { Distribution fo the }\end{array}$ \\
\hline \multirow[t]{2}{*}{ Known existing morbidity } & Present & $45(22.8)$ & $\begin{array}{l}\text { Distribution of the } \\
\text { study subjects }\end{array}$ \\
\hline & Absent & $152(77.2)$ & $\begin{array}{l}\text { Study subjects } \\
\text { according to their }\end{array}$ \\
\hline \multirow[t]{2}{*}{ Addiction } & Present & $47(23.9)$ & socioclinical \\
\hline & Absent & $150(76.1)$ & characteristics \\
\hline \multicolumn{3}{|c|}{ Note(s): *classified as per modified B.G. Prasad scale, January $2019[12]$} & $(n=197)$ \\
\hline
\end{tabular}

\begin{tabular}{llrr}
\hline Risk factor & Category & No. (\%) \\
\hline Age (years) & $<35$ & $47(26.9)$ & \\
& $35-49$ & $87(49.7)$ & \\
Abdominal obesity (in cm) & $>49$ & $41(23.4)$ & \\
(f) = for females & $<80(\mathrm{f}) ; 90(\mathrm{~m})$ & $49(28.0)$ & \\
(m) = for males & $80-89(\mathrm{f}) ; 90-99(\mathrm{~m})$ & $44(25.1)$ & \\
Physical activity & $>=90(\mathrm{f}) ;>=100(\mathrm{~m})$ & $32(1.9)$ & \\
& Vigorous exercise or strenuous at work & $62(35.5)$ & Distribution of study \\
& Moderate exercise at work/home & $73(41.7)$ & participants according \\
& Mild exercise at work/home & $37(21.1)$ & to risk factors of \\
Family history of diabetes & No exercise and sedentary at work/home & $95(54.3)$ & diabetes mellitus type 2 \\
& Both parents nondiabetic & $64(36.6)$ & as per the \\
& One parent diabetic & $16(9.1)$ & IDRS $(n=175)$ \\
\hline
\end{tabular}

(1.129-6.638); $p=0.026]$ and existing known morbidities [AOR = 3.234 (1.453-7.195); $p=0.004$ ] were found to be significantly predicting a high risk of developing diabetes mellitus type 2, placing males and those with comorbidities at higher risk. For this model, the Omnibus test for model coefficient was significant $(\phi=0.000)$, and the Hosmer-Lemeshow test result was insignificant $(p=0.694)$. Nagelkerke's $R^{2}$ and Cox $\&$ Snell $R^{2}$ for the model were 0.134 and 0.101 , respectively. The model could predict correctly in $66.9 \%$ of cases (Table 3 ).

\section{Discussion}

Apart from India and China, countries like the USA, Germany, Italy, Indonesia, Pakistan, Bangladesh, Brazil and Mexico contribute significantly to the worldwide burden of diabetes $[4,13]$. A number of diabetes risk scores have been derived from around the world to detect 
JHR

36,4

742

diabetes cases that have remained undiagnosed, and most of these rely on anthropometric, behavioral and demographic factors [14-17]. Ethnic differences contributing to the risk of developing diabetes make no scoring tool generalizable across the world. The IDRS, derived specifically for the purpose of screening the Indian population, thus becomes a necessary and useful tool in the Indian perspective.

We found a large proportion $(46.8 \% ; n=82)$ of the participants to be at high risk of developing diabetes mellitus type 2 , based on their score obtained with the help of the IDRS; $38.8 \%(n=68)$ were found to be at medium risk, and $14.4 \%(n=25)$ were at low risk of developing the disease. A study from Chennai had $37 \%$ and $45 \%$ of its participants at high risk and moderate risk, respectively [18]. Another similar study from North India revealed $43.3 \%$ of its respondents at moderate risk, while $51.4 \%$ at high risk; a Karnataka-based study found $57.2 \%$ of its participants to be at moderate risk and $33.8 \%$ at high risk [1, 19]. A similar study in rural Karnataka showed $14.84 \%$ to be at high risk and $73.19 \%$ to be at moderate risk; a study from rural Tamil Nadu showed $74.7 \%$ of its study subjects to be at moderate risk and $12.1 \%$ to be at high risk $[10,20]$. The low proportion of high-risk individuals in these two studies could have been because they were performed on a relatively younger population.

Our study and similar other studies as referred above have found that a large proportion of the Indian population, yet to be diagnosed with diabetes, are at a high risk of having the disease. Like in India, nearly two-thirds of total diabetics in most developing countries, have been shown to go undiagnosed, due to lack of awareness, restricted access to healthcare services and inadequately trained healthcare workers; the developed nations have a smaller proportion of their citizens going undiagnosed [2,13]. With cases of diabetes on the constant rise, leading to increasing health expenditure and an ever-growing sick population, early detection of diabetes is a much necessary step. This in turn could help avert the dreaded complications of the disease and thereby reduce the clinical, social and economic burden due to diabetes. Mass screening tools like the IDRS thus become a very important part of India's efforts to fight diabetes, which may be targeted on the vulnerable sections of the population in community-based screening camps. The IDRS offers the scope of providing a cost-effective screening mechanism, which is also practically feasible, something much needed in the Indian scenario.

This study found gender and the presence of existing known comorbidities to be significantly predicting the risk of diabetes. A similar study also found gender to be significantly associated with the risk of diabetes [19]. Males are generally more predisposed towards developing diabetes, as is evident from existing scientific records [21-23]. Comorbidities like hypertension and obesity often go hand in hand with diabetes and the presence of one predisposes an individual to the other [24-26].

Therefore, along with these widespread screening initiatives using tools like the IDRS, extensive campaigns may be undertaken to educate people on the risk factors of diabetes and to encourage people to adopt healthy behaviors in order to curb the risk of developing the

Table 3.

Predictability of risk of diabetes mellitus type 2 with sociodemographic variables by multivariable logistic regression analysis $(n=175)$

\begin{tabular}{|c|c|c|c|c|c|c|}
\hline \multirow[b]{2}{*}{ Variable } & & \multicolumn{2}{|c|}{ Risk of diabetes } & \multirow[b]{2}{*}{ Total } & \multirow[b]{2}{*}{ AOR $(95 \% \mathrm{CI})^{*}$} & \multirow[b]{2}{*}{$p$} \\
\hline & & High risk & Medium and low risk & & & \\
\hline \multirow[t]{2}{*}{ Gender } & Female & 61 & 84 & 145 & Ref & \\
\hline & Male & 21 & 9 & 30 & $0.026(1.129-6.638)$ & 0.026 \\
\hline \multirow{2}{*}{ Addiction } & Absent & 58 & 77 & 135 & Ref & \\
\hline & Present & 24 & 16 & 40 & $1.581(0.735-3.403)$ & 0.241 \\
\hline \multirow[t]{2}{*}{ Co-morbidity } & Absent & 56 & 82 & 138 & $\operatorname{Ref}$ & \\
\hline & Present & 26 & 11 & 37 & $3.234(1.453-7.195)$ & 0.004 \\
\hline
\end{tabular}


disease that can ultimately result in a positive change in the scenario. The need for sidestepping the avoidable risk factors of diabetes may be stressed to people in their growing years at school and college by designing a curriculum focussed on health education. As recommended by the World Health Organization (WHO), in order to discourage people from indulging in harmful practices that predispose them to diabetes, higher taxes may be imposed on sweets and sweetened beverages; detailed labels may be placed on food packaging to educate and motivate them against these[13]. Grassroot health workers [like the ASHAs, who work in villages in India], should be trained to identify symptoms of diabetes, along with the training to perform and interpret basic diagnostic tests. A dedicated diabetes detection kit may be designed and supplied to them for use so that the diabetic status of the person identified to be at risk can be ascertained without delay. The primary health centers may be made fully competent to treat diabetes, ensuring the availability of basic and advanced laboratory tests, along with adequate variety and quantity of antidiabetic medications [13]. The power of mass media like television and radio, which are commonly found in households may be utilized, by design and dissemination of diabetes-related health education communications. Medical colleges may be encouraged to perform high-quality research on diabetes through readily available funds from either the governments or other funding agencies. A dedicated database on diabetes that gets updated real-time, to keep track of the rising number of cases, is also urgently needed.

A strength of this study is that it was field-based. With regards to the limitation of the present study, recall bias could be seen as a limitation as participants who had lost their parents a while back would not be able to recall details of their parents' diabetic status.

\section{Conclusion}

A large number among the Indian population either have diabetes and are yet to be diagnosed or are at a high risk of having the disease. Early detection of the disease can help curtail its complications and reduce its clinical, social and economic burden. Identifying those vulnerable to developing the disease can be useful in order to implement timely primary prevention. A simple mass screening tool like the IDRS, which can be used at the grassroots level by health care and social workers to fight diabetes even in rural and hard to reach areas, thus becomes a very important instrument, especially in a country like India, which is also the second most populous country in the world.

Conflict of Interest: None

\section{References}

1. Acharya AS, Singh A, Dhiman B. Assessment of diabetes risk in an adult population using Indian diabetes risk score in an urban resettlement colony of Delhi. J Assoc Physicians India. 2017; 65(3): 46-51.

2. Mohan V, Deepa R, Deepa M, Somannavar S, Datta M. A simplified Indian Diabetes Risk Score for screening for undiagnosed diabetic subjects. J Assoc Physicians India. 2005; 53: 759-63.

3. Directorate General of Health Services. [cited 2020 Jun 24]. Available from: https://dghs.gov.in/ content/1363_3_NationalProgrammePreventionControl.aspx.

4. Wild S, Roglic G, Green A, Sicree R, King H. Global prevalence of diabetes: estimates for the year 2000 and projections for 2030. Diabetes Care. 2004; 27(5): 1047-53. doi: 10.2337/diacare.27.5.1047.

5. Bhatia T, Oka M, Dharamdasani V, Bhattaccharjee S, Fortwengel G, Limaye V, et al. Type 2 diabetes mellitus: risk evaluation and advice in undergraduate students in Mumbai. Int J Pharm Sci Invent. 2014; 3(4): 37-40.

6. Nandeshwar S, Jamra V, Pal DK. Indian diabetes risk score for screening of undiagnosed diabetic subjects of Bhopal City. Natl J Community Med. 2010; 1(2): 176-7.
Vulnerability towards developing diabetes 
JHR 36,4
7. Patel DN, Shahn MC, Ahir GN, Amin DV, Singh MP. A study on validity of Indian Diabetes Risk Score (MDRF) for screening of diabetes mellitus among the high risk group (policemen) of diabetes mellitus of Bhavnagar city. Innov J Med Health Sci. 2012; 2(5): 109-11.

8. Rao CR, Kulkarni MM, Sathiya NS, Kamath VG, Kamath A, Ballala K, et al. Utility of Indian diabetic risk score (IDRS) in a rural area of coastal Karnataka, India. J Evol Med Dent Sci. 2014; 3(13): 3272-7. doi: 10.14260/jemds/2014/2279.

9. Bhadoria AS, Kasar PK, Toppo NA. Validation of Indian diabetic risk score in diagnosing type 2 diabetes mellitus against high fasting blood sugar levels among adult population of central India. Biomed J. 2015; 38(4): 359-60. doi: 10.4103/2319-4170.143508.

10. Subramani R, Devi U, Shankar U, Stephen, Karthik, Seshadhri, et al. Assessment of risk of type 2 diabetes mellitus among rural population in Tamilnadu by using Indian Diabetic Risk Score. Middle East J Sci Res. 2014; 21(1): 223-5. doi: 10.5829/idosi.mejsr.2014.21.01.21151.

11. Statistics How To. Sample size in statistics (How to find it): excel, Cochran's formula, general tips. [cited 2020 Sep 1]. Available from: https://www.statisticshowto.com/probability-and-statistics/ find-sample-size/.

12. BG Prasad scale. Prasad social classification scale update. [cited 2019 Feb 3]. Available from: http://prasadscaleupdate.weebly.com/.

13. International Diabetes Federation. IDF diabetes atlas. [cited 2020 Sep 15]. Available from: https:// diabetesatlas.org/en/.

14. Glumer C, Carstensen B, Sandbaek A, Lauritzen T, Jorgensen T, Borch-Johnsen K, et al. A Danish diabetes risk score for targeted screening: the Inter99 study. Diabetes Care. 2004; 27(3): 727-33. doi: 10.2337/diacare.27.3.727.

15. Lindstrom J, Tuomilehto J. The diabetes risk score: a practical tool to predict type 2 diabetes risk. Diabetes Care. 2003; 26(3): 725-31. doi: 10.2337/diacare.26.3.725.

16. Nelson KM, Boyko EJ. Predicting impaired glucose tolerance using common clinical information: data from the Third National Health and Nutrition Examination Survey. Diabetes Care. 2003; 26(7): 2058-62. doi: 10.2337/diacare.26.7.2058.

17. Bhowmik B, Siddiquee T, Mujumder A, Ahmed T, Mahtab H, Azad Khan A, et al. Diabetes risk score for identifying cardiometabolic risk factors in adult Bangladeshi population. J Diabetol. 2018; 9(3): 95-101. doi: 10.4103/jod.jod_29_18.

18. Nagalingam S, Sundaramoorthy K, Arumugam B. Screening for diabetes using Indian diabetes risk score. Int J Adv Med. 2016; 3(2): 415-8. doi: 10.18203/2349-3933.ijam20161102.

19. Brahmbhatt KR, Chakraborty T, Gopal C, Shwethashree M, Madappady S, Sowndarya TA, et al. Assessment of risk of type 2 diabetes using simplified Indian Diabetes Risk Score - communitybased cross-sectional study. Int J Med Sci Public Health. 2016; 5(12): 2522-5. doi: 10.5455/ijmsph. 2016.16052016517.

20. Ramaiah R, Jayarama S. Assessment of risk of type 2 diabetes mellitus among rural population in Karnataka by using Indian diabetes risk score. Int J Community Med Public Health. 2017; 4(4): 1056-9. doi: 10.18203/2394-6040.ijcmph20171323.

21. International Institute for Population Sciences [IIPS]; ICF. National family health survey (NFHS-4) 2015-16. Mumbai, India: IIPS; 2017.

22. Logue J, Walker JJ, Colhoun HM, Leese GP, Lindsay RS, McKnight JA, et al. Do men develop type 2 diabetes at lower body mass indices than women? Diabetologia. 2011; 54(12): 3003-6. doi: 10. 1007/s00125-011-2313-3.

23. Nordström A, Hadrévi J, Olsson T, Franks PW, Nordström P. Higher prevalence of type 2 diabetes in men than in women is associated with differences in visceral fat mass. J Clin Endocrinol Metab. 2016; 101(10): 3740-6. doi: 10.1210/jc.2016-1915.

24. Sun P. Recent top 15 comorbid conditions among patients with type 2 diabetes mellitus[mdash] a large national medical records review study. [cited 2020 Jul 14]. Available from: https:// 
professional.diabetes.org/abstract/recent-top-15-comorbid-conditions-among-patients-type-2diabetes-mellitusmdasha-large.

25. Lin PJ, Kent DM, Winn A, Cohen JT, Neumann PJ. Multiple chronic conditions in type 2 diabetes mellitus: prevalence and consequences. Am J Manag Care. 2015; 21(1): e23-34.

26. Valent F, Tillati S, Zanier L. Prevalence and comorbidities of known diabetes in northeastern

Vulnerability towards developing diabetes Italy. J Diabetes Investig. 2013; 4(4): 355-60. doi: 10.1111/jdi.12043.

Corresponding author

Shibaji Gupta can be contacted at: shibaji.dr@gmail.com

For instructions on how to order reprints of this article, please visit our website:

www.emeraldgrouppublishing.com/licensing/reprints.htm

Or contact us for further details: permissions@emeraldinsight.com 